\title{
Noise variance estimation and optimal weight determination for GOCE gravity recovery
}

\author{
J. Kusche \\ Department of Geodesy, Delft University of Technology, Thijsseweg 11, 2629, JA Delft, The Netherlands
}

\begin{abstract}
In the course of level 2 data processing for the GOCE (Gravity Field and Steady-State Ocean Circulation Explorer) satellite mission different streams of level $1 \mathrm{~b}$ data will be merged in a single solution providing the Earth's gravity field, but also state-vector parameters and other quantities. A proper weighting of orbit tracking data, gravity gradiometry data and certain a priori information, usually considered as 'solution constraints', can be expected as crucial for the solution quality. But the a priori stochastic models, based on pre-mission assessment of the expected instrument behaviour, may be over-optimistic or even too pessimistic since they refer to an unprecedented mission with scientific payload never tested in space. One way to derive an optimal weighting scheme on a statistically sound basis while simultaneously validating the stochastic noise levels of the data is by including variance component estimation as a part of the level $1 \mathrm{~b}$ to level 2 data analysis process. The idea is that by applying Monte-Carlo techniques this method can be extended to a large-scale problem like GOCE data analysis, using software modules that already exist or are currently under development. The proposed method has been tested using simulated GOCE orbit trajectories as well as gravity gradiometry data corrupted by colored random noise.
\end{abstract}

Key words. GOCE, gravity field modelling, combination solutions, weight estimation, variance component estimation

\section{Introduction}

As its main product, ESA's Gravity and Steady-State Ocean Circulation Explorer Mission (GOCE) will provide a global model of the Earth's static gravity field (ESA 99). The computation of this model will rely on different level $1 b$ data sets that should be processed ideally in a joint parameter estimation scheme. This includes at least satellite gravity gradiometry data derived from different accelerometer combinations, and satellite-to-satellite tracking data from the GPS

Correspondence to: J. Kusche (J.Kusche@ citg.tudelft.nl) system. Depending on the solution strategy and objectives, airborne data collected over the Earth's polar areas, gravity data or models provided by the current missions CHAMP or GRACE, or adopted a priori constraints on high-degree signal power may be used in the analysis.

Needless to say that the choice of the relative weights for these data sets or models is of vital importance for obtaining the most reliable estimates of the spherical harmonic coefficients, the level 2 product. But also the reliability of the estimated covariance matrix of the gravity field solution will depend on the assumptions about the observation weights. We propose therefore, that a weight optimization process on a stochastic basis should be included in the level $1 \mathrm{~b}$ to level 2 (hereafter abbreviated as L2) data processing. Although a lot of effort is currently put into the pre-mission investigation of error models for the gradiometer instrument and the precise orbit determination (POD) process, and into in-flight calibration and error assessment strategies that apply before arriving at the level $1 \mathrm{~b}$ (Koop et al., 2002), optimization with respect to the weights given by these models within the L2 processing may still improve the final product. At the same time it would serve as a validation of the a priori stochastic models, when real data becomes available. However, also the functional models implemented in the processing will suffer from imperfections, e.g. an aliasing effect might occur due to truncation of the spherical harmonic expansion, and the sensor models will simplify the measurement process. On the one hand, adjusting the stochastic models in a joint estimation process will to a certain extend compensate for this functional mismodelling, on the other hand these effects must be taken into account when interpretating the estimated stochastic parameters.

The method investigated in this paper relies on a statistical basis, i.e. the (almost) unbiased estimation of variance components (VCE), see e.g. Grafarend et al. (1980) or Koch (1990). Looking at the present GOCE data analysis concepts, the main limitation of VCE techniques so far seems the costly and repeated computation of the redundancy contributions of the observation groups, which equal to the trace of the so- 
called observation group influence matrices. The latter is the projection matrix which relates a particular observation group to the corresponding least-squares adjustment residuals; it involves the normal matrix contribution of the particular data set as well as the inverse of the weighted combined normal matrix. For large systems, like those encountered when solving for a high-resolution gravity field model within GOCE L2 processing, this appears prohibitive. Therefore we propose a variant described by Koch and Kusche (2002), which makes use of a stochastic trace estimation technique invented by Girard (1989) and Hutchinson (1990). It has been recently (Kusche, 2003) re-structured and developed further in a Monte-Carlo sense (and called MCVCE), that is, on input for an arbitrary least-square inversion software we use cyclically randomized versions of the original data set where for each observation group in question an artificial noise sequence has to be passed through the inversion. On output, from a comparison of the residuals obtained with the original data and the randomized data the variances are estimated and new weights are chosen in an iterative sense. The main advantage is, that basically an existing software package for solving the L2 inversion problem can be used without modifications.

The material is organized as follows: First, we briefly review the particular iterative VCE algorithm we intend to use. We will explain how this algorithm can be 'mimicked' by embedding a given L2 inversion program in a Monte Carlo framework, that is, by passing artificially generated random data through the inversion. Finally, two simulations under rather simplified condition show how a priori stochastic models for GOCE data can be validated and improved by MCVCE: We consider (1) low-degree gravity field recovery from the GPS orbit determination, where the noise level varies between different (1 day-) orbital arcs, and 2) a simultaneous assessment of the total gradiometer noise power (variance) and the total power of an a priori signal degree variance model. A discussion closes this contribution.

\section{Methodology}

The linear observation model, which shall be adopted here for the joint inversion of $p$ independant observation groups collected from GOCE, reads

$\boldsymbol{X}_{i} \boldsymbol{\beta}=\boldsymbol{y}_{i}+\boldsymbol{e}_{i} \quad i \in\{1, \ldots, p\}$.

The $n_{i} \times u$ matrices $\boldsymbol{X}_{i}$ are the design matrices, the $u \times 1$ vector $\boldsymbol{\beta}$ represents the unknown spherical harmonic coefficients plus additional unknowns such as state-vector parameters, the $n_{i} \times 1$ vectors $\boldsymbol{y}_{i}$ contain the observations, and the $n_{i} \times 1$ vectors $\boldsymbol{e}_{i}$ the stochastic observation errors. The observation vectors may be internally correlated (the noise may be coloured) but are assumed to be uncorrelated with respect to each other. Moreover, we assume that the covariance matrices of these observation groups are known a priori only up to some scaling factors, the $p$ noise levels or variance components $\sigma_{i}^{2}$ :

$E\left(\boldsymbol{e}_{i} \boldsymbol{e}_{j}^{\prime}\right)=0 \quad E\left(\boldsymbol{e}_{i} \boldsymbol{e}_{i}^{\prime}\right)=\sigma_{i}^{2} \boldsymbol{P}_{i}^{-1}$

This means, that the total number of unknowns in our problem is $u+p$. The actual number $p$ of these subdivisions of the overall GOCE data set, corresponding to the number of degrees of freedom in the stochastic model, will be a part of the solution strategy and as such subject to individual considerations and growing experience in the course of the L2 processing phase. If only the weighting between GPS-derived precise orbit (POD) data and gradiometry data as a whole is considered, $p$ equals to 2 . If, in addition, we allow for adjusting the weight of a priori information (contraining the very high degrees), $p$ equals to 3 . If one breaks off the stochastic model by allowing for varying noise levels in time, e.g. after orbit manoeuvres, internal re-calibrations, or between orbital arcs, and in space, e.g. between the different gradiometer components, $p$ might become reasonably large.

The estimation of variance components $\sigma_{i}^{2}$ (Grafarend et al., 1980; Koch 1990) leads generally to a coupled iterative process, since both the calculation of the so-called group redundancies $r_{i}$ (see below, step 4) as well as of the residuals $\hat{\boldsymbol{e}}_{i}$ involve knowledge of all variance levels $\sigma_{j}^{2}, j=1 \ldots p$. The strategy is then:

1. Select $p$ start values for the noise levels $\sigma_{i}^{2(0)}$

2. Compute a solution $\hat{\boldsymbol{\beta}}^{(k)}$ from the combined normals (we assume an operation $\boldsymbol{P}_{i} \boldsymbol{u}_{i}$ may be replaced by a filtering procedure)

$$
\left(\sum_{i=1}^{p} \frac{1}{\sigma_{i}^{2^{(k)}}} \boldsymbol{X}_{i}^{\prime} \boldsymbol{P}_{i} \boldsymbol{X}_{i}\right) \hat{\boldsymbol{\beta}}^{(k)}=\sum_{i=1}^{p} \frac{1}{\sigma_{i}^{2^{(k)}}} \boldsymbol{X}_{i}^{\prime} \boldsymbol{P}_{i} \boldsymbol{y}_{i}
$$

3. Compute the $p$ residual vectors $\hat{\boldsymbol{e}}_{i}^{(k)}=\boldsymbol{X}_{i} \hat{\boldsymbol{\beta}}^{(k)}-\boldsymbol{y}_{i}$

4. Compute the $p$ group redundancy numbers, $r_{i}{ }^{(k)}$, from

$$
\begin{aligned}
& r_{i}^{(k)}= \\
& n_{i}-\frac{1}{\sigma_{i}^{2^{(k)}}} \operatorname{trace}\left(\left(\sum_{i=1}^{p} \frac{1}{\sigma_{i}^{2^{(k)}}} \boldsymbol{X}_{i}^{\prime} \boldsymbol{P}_{i} \boldsymbol{X}_{i}\right)^{-1} \boldsymbol{X}_{i}^{\prime} \boldsymbol{P}_{i} \boldsymbol{X}_{i}\right)
\end{aligned}
$$

5. Determine $p$ new variance components, and continue with step 2 until convergence

$$
\sigma_{i}^{2(k+1)}=\frac{\hat{\boldsymbol{e}}_{i}^{\prime(k)} \boldsymbol{P}_{i} \hat{\boldsymbol{e}}_{i}^{(k)}}{r_{i}^{(k)}}
$$

How many iterations are required generally depends on how good the functional and the stochastic models fit the observations, and is therefore difficult to predict in advance. 
The group redundancy numbers are key quantities in the assessment of combination solutions and their information content: By their definition, $r_{i}^{(k)}=\operatorname{trace}\left(\partial \hat{\boldsymbol{e}}_{i}^{(k)} / \partial \boldsymbol{y}_{i}\right)$, they quantify the overall contribution of a certain observation group to the final solution. But step 4 obviously poses a problem for GOCE L2 processing: The computation of the related matrix requires repeated solutions of the normal system with the columns of the $i$-th individual normal matrix as righthand side. This would probably require major modifications in the inversion software, and it is doubtful that this operation can be performed in reasonable time even with supercomputers. Therefore, in the following we aim at obtaining a computational cheap Monte Carlo estimate of the redundancy numbers.

\section{Monte Carlo implementation}

A Monte Carlo implementation of the computation of the group redundancy numbers, based on trace estimation techniques, has been originally proposed by Koch and Kusche (2002), and elaborated further in Kusche (2003). We skip the details here. One way is to replace the $p$ contribution measures $r_{i}^{(k)}$ in step 4 by the estimates $\hat{r}_{i}^{(k)}$, which follow from

$\hat{r}_{i}^{(k)}=n_{i}-\frac{1}{\sigma_{i}^{4^{(k)}}} \boldsymbol{w}_{i}^{\prime} \boldsymbol{P}_{i} \boldsymbol{X}_{i} \hat{\boldsymbol{p}}_{i}^{(k)}$.

Here $\boldsymbol{w}_{i}$ is an artificially generated $n_{i} \times 1$ random vector with zero expectation and variance-covariance matrix $D\left(\boldsymbol{w}_{i}\right)=$ $\frac{\sigma_{i}^{2^{(k)}}}{\sigma_{i}^{2(0)}} \sigma_{i}^{2^{(0)}} \boldsymbol{P}_{i}^{-1}$, thus possessing the same stochastic first and second moments as expected for the (true but unknown) error vector $\boldsymbol{e}_{i}$. The vector $\hat{\boldsymbol{p}}_{i}^{(k)}$ is nothing else but the parameter vector $\hat{\boldsymbol{\beta}}$ obtained when replacing $\boldsymbol{y}_{i}$ by the artificial random vector $\boldsymbol{w}_{i}$ and all other observation groups $\boldsymbol{y}_{j}, j \neq i$, simply by zeroes. The Fig. 1 points out the principle: After step 2 (the inversion software solves from all available data sets for the gravity unknowns, given an initial weighting scheme), one has to run again the inversion for each of the $p$ data sets with the same normal or design matrix but with different right-hand sides $\boldsymbol{X}_{i}^{\prime} \boldsymbol{P}_{i} \boldsymbol{w}_{i}$. Since each of these righthand sides consists of zeroes for $p-1$ data sets, the overall numerics to be added (within one iteration of the process) is one re-computation of a full right-hand side vector, and $p$ solutions of the normal equations for varying right-hand sides. Using these solutions, $p$ data synthesis operations have to be added to obtain the $p$ variance estimates and a new weighting scheme.

\section{Numerical studies}

4.1 Noise variance estimation within long-wavelength gravity field recovery from the GPS-POD

The GPS receiver aboard GOCE plays a multiple role; it enables a high-precision orbit determination allowing the gra- diometer data to be processed without estimation of orbit errors, and it serves for recovery of the long-to-medium part of the gravity field. The POD is expected to be at the $\mathrm{cm}$ accuracy level (Visser and van den IJssel, 2000).

In this study a simulated 10 days GOCE orbit solution has been used as pseudo observation set, i.e. cartesian $x$, $y$, and $z$ coordinates in an Earth-centered quasi-inertial reference frame, split up into $p=10$ data sets (orbital arcs) of 1 day each. In an adjustment for gravity parameters $\boldsymbol{\beta}=\left(\delta c_{02}, \delta c_{12}, \delta s_{12}, \ldots\right)^{T}$ (that is, the difference of the harmonic coefficients between the 'true' model OSU91a and the adopted initial model JGM-3) as well as for 10 sets of state-vector epoch parameters $\boldsymbol{\beta}_{i}=\left(x, y, z, v_{x}, v_{y}, v_{z}\right)^{T}$, $i=1 \ldots 10$, the partial derivatives of the satellite positions with respect to the unknowns have been obtained by numerical integration of the variational equations. The OSU91a model has been used complete up to degree 50 for the orbit generation. One has to note that the parameter estimation process actually has to be iterated due to linearization errors; however these errors are systematic but small for degree 50 and no re-computation of the partials was applied. Also no regularization was imposed on the estimation process. Of course, these considerations should be revised when higher resolutions will be taken into account. Simulated noise-free data and partials files, computed from the GEODYN II orbit determination software (McCarthy et al., 1993) have been kindly provided by $\mathrm{P}$. Visser. A detailed description is given in (Visser et al., 2001), where this data set has been used in a comparative study on the quality of different recovery methods. At the time of writing, it is indeed expected that the variational approach will be followed in the derivation of the official ESA level-2 gravity field product from the GPS POD. We added a generated colored noise sequence to these data, whose power spectral density (PSD) model shows peaks of $(2 \mathrm{~cm} / 1.5 \mathrm{~cm} / 0.8 \mathrm{~cm})$ at the $1 \mathrm{cpr}$ frequency in along/cross/radial orbit direction, and remains flat elsewhere.

For the inversion, these pseudo-observed coordinates $x, y$, and $z$ were assumed as uncorrelated in time as well as with respect to each other (thus both assumptions causing stochastic model errors), and an unknown variance component $\sigma_{i}^{2}$ has been assigned to each orbital arc. For all experiments we assumed equal start values $\sigma_{i}^{2}=(1 \mathrm{~cm})^{2}$ for the first iteration. Results are given for the estimated common gravity parameters $\hat{\boldsymbol{\beta}}$, expressed in terms of geoid height errors, and for the estimated variance components.

In a first simulation run, we scaled the generated noise sequences for each arc individually by a random factor with expectation one and sigma 0.5 , thus simulating fluctuations of the noise level. Geoid errors and the range of the estimated variance levels are shown in Table 1. Clearly, such moderate noise level fluctuations have only minor influence on the gravity field solution. But nevertheless, a few iterations of MCVCE could improve the solution somewhat in terms of maximum geoid errors (being located at the polar areas due to the lack of measurements). The estimated range of variances give a good validation of the simulated fluctuation. 


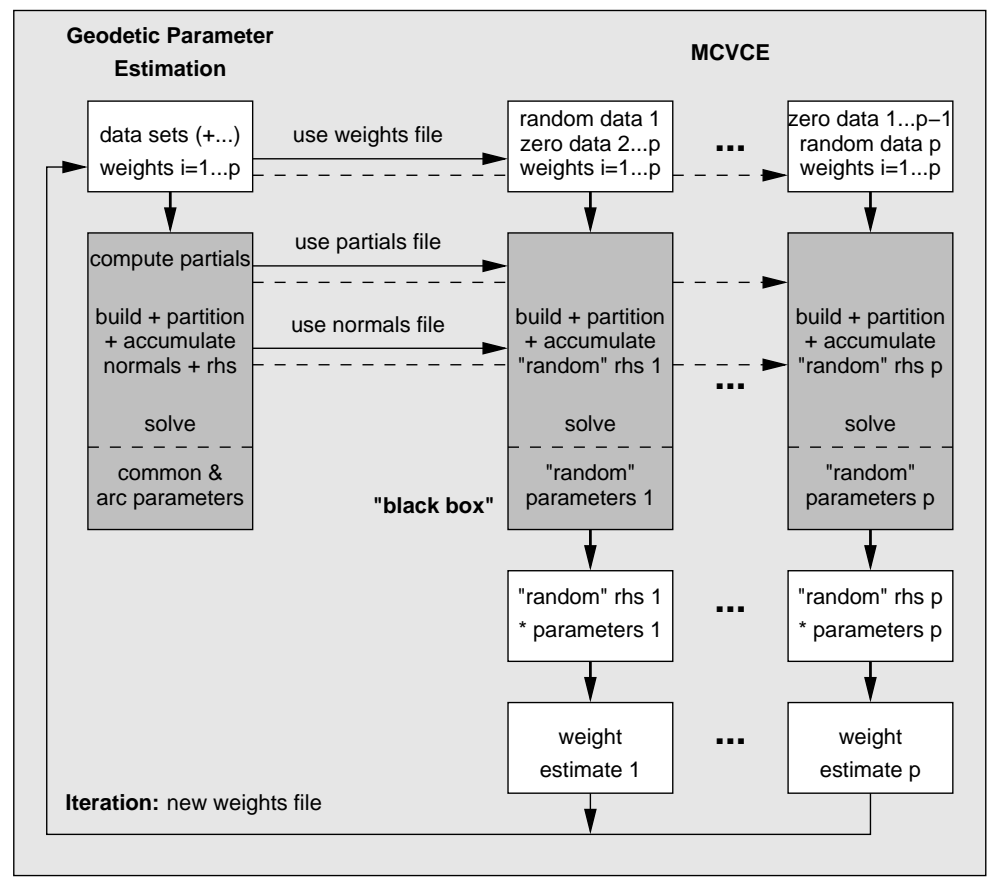

Fig. 1. Modular use of inversion software within MCVCE software.

Table 1. Results for the first case. Noise levels vary between the arcs with $0.5 \mathrm{~cm}$ standard deviation

\begin{tabular}{ccccc}
\hline \multirow{2}{*}{ case } & \multicolumn{3}{c}{ Geoid errors [cm] } & \\
\cline { 2 - 4 } & $\max$ & rms & average & estimated $\hat{\sigma}_{i}[\mathrm{~cm}]$ \\
\hline equal weights & 105.3 & 11.0 & 16.2 & 1 \\
\hline 1st iteration & 104.3 & 11.1 & 16.4 & $0.8 \ldots 1.4$ \\
\hline 5th iteration & 100.9 & 11.0 & 16.3 & $0.7 \ldots 1.7$ \\
\hline convergence & 100.3 & 10.9 & 16.2 & $0.6 \ldots 1.8$ \\
\hline
\end{tabular}

Afterwards, we repeated the same experiment but for two arcs the simulated noise level was now doubled with respect to the original $(2 \mathrm{~cm} / 1.5 \mathrm{~cm} / 0.8 \mathrm{~cm})$ noise sequence, see table 2. The two 'bad' arcs (20\% of the data!) in fact cause strongly increased geoid errors, when no downweighting takes place at all. After downweighting in the course of the MCVCE iteration, these errors are damped reasonably.

4.2 Assessing total power of noise and of signal-variance constraints in a gradiometry-only solution

The performance of MCVCE has been investigated, in a second study, for its ability of validating the gradiometer total noise power in a full-scale simulation, complete until degree and order 300 . One has to note that for this high resolution some kind of regularization is indispensible. In the present study this is achieved by introducing different Kaula-type
Table 2. Results for the first case. For two arcs the noise level has been doubled

\begin{tabular}{ccccc}
\hline \multirow{2}{*}{ case } & \multicolumn{3}{c}{ Geoid errors [cm] } & \\
\cline { 2 - 4 } & $\max$ & rms & average & estimated $\hat{\sigma}_{i}[\mathrm{~cm}]$ \\
\hline equal weights & 158.4 & 16.6 & 24.1 & 1 \\
\hline 1st iteration & 132.6 & 13.6 & 20.1 & $1.4 \ldots 2.2$ \\
\hline 5th iteration & 114.0 & 12.7 & 18.3 & $0.9 \ldots 2.8$ \\
\hline convergence & 107.7 & 12.4 & 17.8 & $0.8 \ldots 2.8$
\end{tabular}

'weak' constraints for the signal power of the potential or of derivatives; 'weak' means that the total variance of these a priori models leaves to be determined from the analysis of the data. With other words, a variance factor for the a priori model has to estimated.

A circular orbit, almost repeat with 961 orbit revolutions during 59.8 nodal days, has been generated. The difference between OSU91a and GRS80 defines the 'true' disturbing potential to be recovered. Along the orbit second radial derivatives of the disturbing potential were generated at known positions with a sampling rate of 5 seconds, which gives about one million observations. These were corrupted by a coloured noise, generated from a power spectral density function with a flat spectrum of $9 \mathrm{mE}^{2} / \mathrm{Hz}$ between $0.005 \mathrm{~Hz}$ and $0.1 \mathrm{~Hz}$ and a $1 / f^{2}$ behaviour between $3.7 \cdot 10^{-4} \mathrm{~Hz}$ and $0.005 \mathrm{~Hz}$. The time-wise approach was followed in the com- 
Table 3. Results for the second case

\begin{tabular}{cccc}
\hline \multirow{2}{*}{ case } & \multicolumn{3}{c}{ estimated $\hat{\sigma}_{1}, \hat{\sigma}_{2}[-],[-]$} \\
\cline { 2 - 4 } & constraint $l^{4}$ & constraint $l^{6}$ & constraint $l^{8}$ \\
\hline initial weights & 1,1 & 1,1 & 1,1 \\
\hline 1st iteration & $1.006,0.981$ & $0.994,146$ & $1.016,22100$ \\
\hline 5th iteration & $1.007,0.982$ & $1.028,135$ & $1.050,20380$ \\
\hline convergence & $1.007,0.982$ & $1.028,135$ & $1.050,20370$ \\
\hline
\end{tabular}

putation of the normal equations, yielding a strictly blockdiagonal normal matrix. Coloured noise has been taken into account in MCVCE by replacing operations $\boldsymbol{v}=\boldsymbol{P} \boldsymbol{u}$ by an ARMA-filter, $v_{n}=u_{n}-\sum_{k=1}^{p} a_{p, k} v_{n-k}+\sum_{l=1}^{q} b_{q, i} u_{n-i}$. In the variance-component estimation, this filter implementation can be of low order; we found $p=q=2$ sufficient.

For regularization, we added Kaula-type matrices $\boldsymbol{K}$ to the normal equations; this means, with entries of order $\sim l^{4}$, $l^{6}$ (first order derivative regularization), or $l^{8}$ (second order derivative regularization). The total variance $\sigma_{1}^{2}$ of the gradiometer observations, as well as a variance factor $\sigma_{2}^{2}$ for the signal constraint matrix, has been left open within the analysis for determination by MCVCE. That is, the normal equations in step 2 of the algorithm of section 2 read explicitly

$$
\left(\frac{1}{\sigma_{1}^{2^{(k)}}} \boldsymbol{X}^{\prime} \boldsymbol{P} \boldsymbol{X}+\frac{1}{\sigma_{2}^{2^{(k)}}} \boldsymbol{K}\right) \hat{\boldsymbol{\beta}}^{(k)}=\frac{1}{\sigma_{1}^{2^{(k)}}} \boldsymbol{X}^{\prime} \boldsymbol{P} \boldsymbol{y}
$$

The geoid rms errors are of the order $16 \mathrm{~cm}$ for all cases, when excluding the polar areas. This reflects the observation that the quality of the gravity field solution is not very sensitive with respect to the choice of the regularization matrix. But looking at Table 3 we conclude that if the aim is to validate the gradiometer noise level, the constraint implemented in the regularization matrix should not deviate too much from the power (degree variances) of the Earth's true gravity field. This could be expected since in the stochastic interpretation $\sigma_{2}^{2} \boldsymbol{K}$ takes the role of the a priori variance-covariance matrix of the spherical harmonic coefficients. Ideally, $\hat{\sigma}_{1}$ would be estimated to 1 , meaning that the a priori stochastic model that has been used both for simulation and for data analysis, is perfectly validated. Small deviations may be related to imperfections in the filter design.

\section{Discussion}

A method has been proposed for validating the variance levels for the different GOCE observation types, and based on this, for the determination of an optimal weighting scheme. The method treats all observations as input within a joint parameter estimation, thus without leaving certain data out for independent validation. It relies basically on the well-known and powerful method of variance-component estimation, recast in a Monte Carlo framework. As a consequence, it can be used without re-coding existing L2 inversion software. However, numerical studies so far concern only very specific test cases, and experience has still to be gained. A point of concern might be that (multiplicative) variance components can only account for the total power of a particular stochastic model, relying on the a priori structure of the variancecovariance matrix for this type of observations. In the present form it is therefore not possible to relate an estimated variance level to a specific bandwidth in a given power spectral density (PSD) model; that is to improve the PSD model itself apart from a simple re-scaling. To this end, the joint estimation scheme would have to be further extended for covariance components, each of which facilitating an additional degree of freedom in the (time-wise) correlation structure of an observation type. However, whereas the theory and application of variance-covariance-component estimation is wellinvestigated, efficient Monte Carlo type algorithms still have to be developed.

Acknowledgements. We thank Pieter Visser from DEOS for providing us with simulated GOCE orbit data and partials files. J.-P. Barriot and an anonymous reviewer gave helpful remarks that improved the quality of the manuscript.

\section{References}

ESA: Gravity Field and Steady-State Ocean Circulation Mission. Reports for Mission Selection, ESA SP-1233(1), ESTEC, Noordwijk, 1999.

Grafarend, E. W., Kleusberg, A., and Schaffrin, B.: An introduction to the variance-covariance component estimation of Helmert type, Zeitschrift für Vermessungswesen, 105, 161-180, 1980.

Koch, K.-R.: Bayesian Inference with Geodetic Applications, Springer, Berlin, 1990.

Koch, K.-R. and Kusche, J.: Regularization of geopotential determination from satellite data by variance components, J. Geodesy, 76, 259-268, 2002.

Koop, R., Bouman, J., Schrama, E. J. O., and Visser, P.: Calibration and error assessment of GOCE data, in: Vistas for Geodesy in the New Millenium, (Eds) Adam, J. and Schwarz, K.-P., Springer, 2002.

Kusche, J.: A Monte Carlo Technique for Weight Estimation in Satellite Geodesy, J. Geodesy, 76, 641-652, 2003.

McCarthy, J. J., Rowton, S., Moore, D., Pavlis, D. E., Luthcke, S. B., and Tsaoussi, L. S.: GEODYN II Systems Description, NASA Goddard Space Flight Center, Greenbelt, Maryland, 1993.

Visser, P. and van den IJssel, J.: GPS-based precise orbit determination of the very low Earth-orbiting gravity mission GOCE, J. Geodesy, 74, 590-602, 2000.

Visser, P., van den IJssel, J., Koop, R., and Klees, R.: Exploring gravity field determination from orbit perturbations of the european gravity mission GOCE, J. Geodesy, 75, 89-98, 2001. 